\title{
The role of focal adhesion kinase in mammary stem cell and tumorigenesis
}

\author{
Ming Luo ${ }^{1}$, Tamas Nagy ${ }^{1}$, Jun-Lin Guan ${ }^{1,2}$ \\ ${ }^{I}$ Division of Molecular Medicine and Genetics, Dept of Internal Medicine, University of Michigan Medical School, Ann Arbor, MI \\ 48109, USA; ' Shanghai Institute of Biochemistry and Cell Biology, SIBS, Shanghai, China
}

An emerging concept in breast tumorigenesis is that mammary stem cells (MaSCs) with dysregulated selfrenewal ability are likely to be the critical cells that propagate tumors. Thus, the characterization of key signaling proteins that regulate MaSC self-renewal and maintenance will be crucial for the development of novel treatment strategies targeting the mammary cancer stem cell (MaCSC) pool. Focal adhesion kinase (FAK), an intracellular tyrosine kinase integrating signals from integrin and growth-factor receptors, plays key roles in cell adhesion, survival, proliferation, migration and angiogenesis. Elevated FAK expression and activation have been found in different stages of breast cancer, yet the role of intrinsic FAK to promote mammary tumorigenesis in vivo remains to be defined. Recent identification of both alpha6 and beta1 integrins as surface markers of mouse MaSCs suggests that FAK-mediated integrin signaling may play an important role in these cells. Consistent with this, we found increased FAK activation in the mouse and human mammary epithelial cells (MaECs) enriched in MaSCs and MaCSC using independent markers. To investigate the role and mechanisms of FAK and its associated signaling pathways in MaSCs, we have created FAK conditional knockout mice (MFCKO) in the mammary gland by using Cre-loxP approach. Analysis of primary MaECs from MFCKO and control mice indicated that inactivated of FAK significantly decreased the MaSCs in the MFCKO mice. Using a mouse breast cancer model, we further showed that the decrease of the MaSCs in the MFCKO mice correlated with a suppression in the development of breast cancer in these mice. These results suggested an important role for FAK signaling pathways in the regulation of MaSCs self renewal and breast cancer development.

Keywords: FAK, mammary stem cells, signal transduction, breast cancer

Cell Research (2008) 18:s98. doi: 10.1038/cr.2008.188; published online 4 August 2008

Correspondence: Jun-Lin Guan

E-mail: jlguan@umich.edu

Jun-Lin Guan obtained his PhD from University of California at San Diego in 1987 and then joined Center for Cancer Research at M.I.T. for his postdoctoral studies from 1987 to 1991 . He was a faculty member at Cornell University from 1991 to 2006. Currently, Dr Guan is a Professor in the Division of Molecular Medicine and Genetics, Dept of Internal Medicine, University of Michigan Medical School. The goals of research programs in Dr Guan's laboratory are to understand fundamental principles of cell signaling in the regulation of basic cellular functions in normal cell and developmental processes and to determine how disruption of the normal signaling pathways either by genetic mutations or environmental insults may lead to diseases such as cancer. 